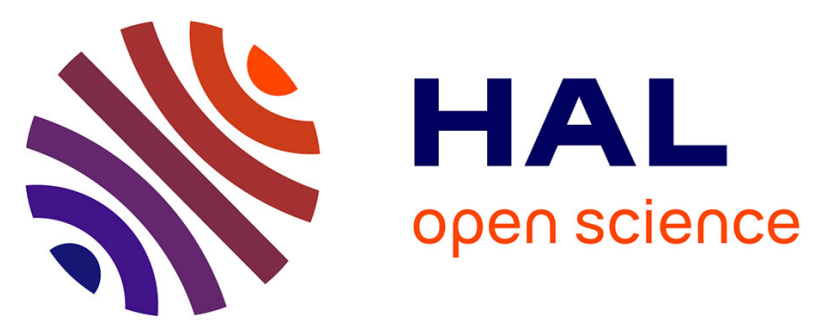

\title{
PRESS-MAG-O: A new facility to probe materials and phenomena under extreme conditions
}

Daniele Di Gioacchino, Paolo Tripodi, Augusto Marcelli, Mariangela Cestelli Guidi, Massimo Piccinini, Paolo Postorino, Daniele Di Castro, Emanuele Arcangeletti

\section{To cite this version:}

Daniele Di Gioacchino, Paolo Tripodi, Augusto Marcelli, Mariangela Cestelli Guidi, Massimo Piccinini, et al.. PRESS-MAG-O: A new facility to probe materials and phenomena under extreme conditions. Journal of Physics and Chemistry of Solids, 2009, 69 (9), pp.2213. 10.1016/j.jpcs.2008.03.036 . hal00565439

\section{HAL Id: hal-00565439 \\ https://hal.science/hal-00565439}

Submitted on 13 Feb 2011

HAL is a multi-disciplinary open access archive for the deposit and dissemination of scientific research documents, whether they are published or not. The documents may come from teaching and research institutions in France or abroad, or from public or private research centers.
L'archive ouverte pluridisciplinaire $\mathbf{H A L}$, est destinée au dépôt et à la diffusion de documents scientifiques de niveau recherche, publiés ou non, émanant des établissements d'enseignement et de recherche français ou étrangers, des laboratoires publics ou privés. 


\section{Author's Accepted Manuscript}

PRESS-MAG-O: A new facility to probe materials and phenomena under extreme conditions

Daniele Di Gioacchino, Paolo Tripodi, Augusto

Marcelli, Mariangela Cestelli Guidi, Massimo Piccinini, Paolo Postorino, Daniele Di Castro, Emanuele Arcangeletti

PII: $\quad$ S0022-3697(08)00106-6

DOI: $\quad$ doi:10.1016/j.jpcs.2008.03.036

Reference: $\quad$ PCS 5437

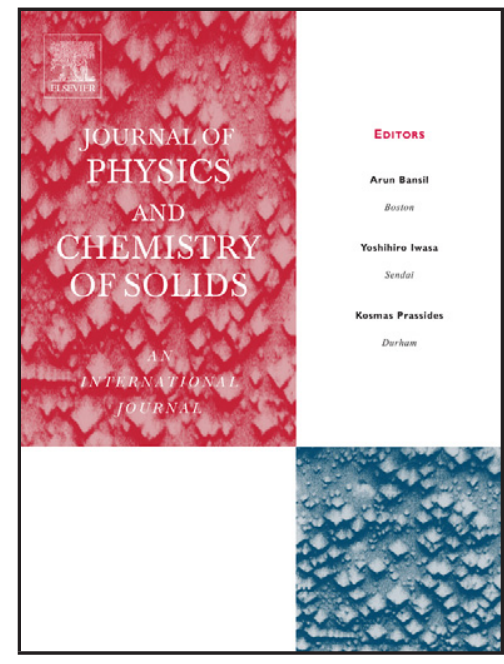

www.elsevier.com/locate/jpcs

To appear in: Journal of Physics and Chemistry of Solids

Cite this article as: Daniele Di Gioacchino, Paolo Tripodi, Augusto Marcelli, Mariangela Cestelli Guidi, Massimo Piccinini, Paolo Postorino, Daniele Di Castro and Emanuele Arcangeletti, PRESS-MAG-O: A new facility to probe materials and phenomena under extreme conditions, Journal of Physics and Chemistry of Solids (2008), doi:10.1016/j.jpcs.2008.03.036

This is a PDF file of an unedited manuscript that has been accepted for publication. As a service to our customers we are providing this early version of the manuscript. The manuscript will undergo copyediting, typesetting, and review of the resulting galley proof before it is published in its final citable form. Please note that during the production process errors may be discovered which could affect the content, and all legal disclaimers that apply to the journal pertain. 


\title{
PRESS-MAG-O: a new facility to probe materials and phenomena under extreme conditions
}

\author{
Daniele Di Gioacchino ${ }^{1}$, Paolo Tripodi ${ }^{1,2}$, Augusto Marcelli ${ }^{1}$, Mariangela Cestelli Guidi ${ }^{1}$, \\ Massimo Piccinini ${ }^{1,4}$, Paolo Postorino ${ }^{3}$, Daniele Di Castro ${ }^{3}$, Emanuele Arcangeletti ${ }^{3}$ \\ 1- INFN-LNF, National Institute of Nuclear Physics, Via Enrico Fermi, 40 - 00044 Frascati (Rome), Italy \\ 2- H.E.R.A. Hydrogen Energy Research Agency, Corso della Repubblica, 448 - 00049 Velletri (Rome), Italy \\ 3- INFM-CNR-Coherentia, Univ. di Roma 'La Sapienza', Dip. di Fisica, P.le Aldo Moro, 4 - 00100 Rome, Italy \\ 4- Università Roma Tre, Dip. di Scienze Geologiche, L.go S. Leonardo Murialdo, 1 - 00146 Rome, Italy
}

\begin{abstract}
In the recent years several experiments performed under high magnetic fields [HMF], at high pressure [HP] and/or at low temperature [LT] have led to spectacular discoveries in condensed matter. In many new systems, although challenging, it is strategic to perform a Magneto-Optical analysis, to investigate the phonon behavior in the far infrared (IR) domain. By combining HMF and HP in a wide temperature (T) range to perform simultaneously IR magneto-optics and ac-magneto-dynamic experiments, it will be possible to achieve unique information on systems and/or new phenomena, almost impossible to obtain with standard spectroscopic methods. Here we present PRESS-MAG-O, a new facility under construction that will perform HP experiments under HMF in a wide $T$ range. The system is expected to be operational by the end of 2007 and will be tested at SINBAD, the IR synchrotron radiation beamline operational since 2001 at DAФNE, the storage ring of the INFN Frascati National Laboratory (LNF). While for IR experiments an interferometer will be used, for the magneto-dynamic experiments a SQUID magnetometer in the $10 \mathrm{~Hz}-2 \mathrm{KHz}$ frequency range will be utilized. $\mathrm{HP}$ will be applied to samples by a Cu-Be diamond anvil cell (DAC), so that the device will be able to collect FTIR spectra and high harmonic ac susceptibility data in a dc magnetic field up to $8 \mathrm{~T}$ and to about $20 \mathrm{GPa}$ in a wide temperature range $(4.2-200 \mathrm{~K})$.
\end{abstract}

Phase Transition, High Pressure, High Harmonic Magnetic ac susceptibility measurements, IR spectroscopy.

\section{INTRODUCTION}

In the last years experiments performed on materials under extreme conditions of high magnetic field, high pressure, at ultra low temperature have led to the spectacular discovery of a great deal of new phenomena as: fractional Quantum Hall Effect $(\mathrm{HMF}+\mathrm{LT})$, ferromagnetic superconductivity in $\mathrm{UGe}_{2}(\mathrm{LT}, \mathrm{HP})$, superconductivity in $\mathrm{S}$ and $\mathrm{Li}$ with largest $\mathrm{T}_{\mathrm{c}}(\mathrm{HP})$ among the elemental superconductors $\mathrm{K}$ [1-3], second triple point in liquid phosphorus, metal to insulator transition in a Mott insulator $\mathrm{BaVS}_{3}[4]$ and a Kondo Insulator $\mathrm{FeSi}_{1-\mathrm{x}} \mathrm{Ge}_{\mathrm{x}}$ [5].

By tuning crystal lattice structure, HP allows changing electronic and magnetic properties of materials [6]. The possibility to follow magnetic properties under pressure, using ac magnetic multi-harmonic susceptibility, allows measuring the critical temperature of new phases and electron dynamic changes. Moreover, IR spectroscopy may investigate different material properties such as phonons and thermo-chemical characteristics, collective modes in correlated systems, etc. In this contest the high brilliance of the synchrotron IR radiation is ideal for HP investigations because with its small beam it may fit at the best the small sample area inside a DAC.

Here we present the status of a new apparatus we are developing, called PRESS-MAG-O, where the simultaneous collection of both ac multi-harmonic susceptibility and FTIR data will be performed under extreme conditions of HP, magnetic field and temperature. PRESS-MAG-O is an acronym correlated to the sentence: "Collective phenomena in solid state physics can be trigged by PRESSure, high magnetic dc field and temperature and can be characterized by MAGneto dynamic and magneto Optical techniques" used at the presentation of the project to the $5^{\text {th }}$ Scientific Committee of the INFN held in a national meeting held in Assisi in 2004. This unique apparatus will allow accurate investigations of exotic materials and quantum phenomena.

\section{PRESS-MAG-O APPARATUS}

Using the superconducting magnet and the high-pressure cell severe experimental conditions can be applied to a sample. The induced physical changes will be measured by combining magneto-optical spectroscopy and multi harmonic magneto- 
dynamic experiments. The control of the experiments is a demanding task that required the development of a novel apparatus whose characteristics are summarized below.

\section{A) Cryostat and split magnet}

An $8 \mathrm{~T}$ superconducting magnet with a split coil and four radial access ports has been manufactured by American Magnetics Inc. (USA). The magnet (showed in Fig.1) will be placed in a cryostat, manufactured by DG-Technology Service (Parma, Italy), equipped by 'ultra flex GARLOK' gaskets showed in Fig.2. The system is designed with the magnetic field perpendicular to the light propagation vector $\left(\mathrm{k}_{\mathrm{IR}}\right)$ (Voight geometry). The instrument has a liquid helium (LHe) reservoir around the magnet and an internal 'sample-insert' device, containing the DAC and the magnetometer, capable to perform static sample cooling via a cold finger on the bottom side. Two cold shields, one at liquid nitrogen $\left(\mathrm{LN}_{2}\right)$ temperature and one at LHe vapor temperature, surround the LHe reservoir to prevent thermal radiation. In order to allow transmission of the IR radiation and to limit the absorption of the IR radiation, the entire device has been designed with only two thin wedged diamond windows of $15 \mathrm{~mm}$ of diameter set along the light path in addition to the two 'anvils' of the diamond cell.

\section{B) Diamond Anvil Cell}

To produce high pressure we manufactured a miniature DAC [7] (Fig.3). AC susceptibility measurements of extremely small volume samples $\left(\sim 1 \times 10^{-7} \mathrm{~cm}^{3}\right)$ at high pressure are a complex assignment also because of the presence of a possible magnetic background inside the cell. To reduce the background and any additional magnetic noise, a non-magnetic $2 \%$ Be$\mathrm{Cu}$ alloy for the cell and the gaskets has been selected. Moreover, to interface the $\mathrm{Cu}$-Be and the anvils backing cylinders with conic holes made by non-magnetic $\mathrm{SiC}-\delta$ phase has been manufactured. $\mathrm{SiC}$ will replace the standard superconducting tungsten carbide that exhibits a $\mathrm{T}_{\mathrm{c}} \sim 4 \mathrm{~K}$ and superconducting fluctuations up to $6 \mathrm{~K}$ [8], making difficult or impossible investigations of superconducting or other quantum transition in this temperature range.

\section{C) ac-gradiometer and SQUID}

In our case a very weak magnetic signal induced in a sample by the superconducting magnet in the presence of a high background noise has to be detected. In fact the extremely small volume of the DAC cell respect to the large pick-up coil dimensions gives an extremely low fill factor of the pick-up coils. A SQUID device coupled to a gradiometer, employed as a flux transformer in the high magnetic field probing the sample response, is the best instrument available to fulfill the challenging experimental conditions of our system. In Fig.4, we show both the layout of the planar ac micro-gradiometer designed to work inside the DAC and a photograph of the pick-up coils patterned on a Si wafer. In the present design the pick-up coil will be set above the gasket and two $\mathrm{Nb}$ thin film loop sensors in the integrated planar micro-gradiometer have been realized by lithography technique on a mono-crystal silicon wafer. The geometrical configuration has been optimized to fit the gasket position around the diamonds of the DAC.

\section{D) DAC/gradiometer sample insert}

The ac magnetic multi-harmonic susceptibility response of the sample excited by an ac drive field will be detected by the SQUID micro-gradiometer. For this reason a $\mathrm{Cu}$-Be removable sample insert showed in Fig. 5 has been manufactured. The insert will have a thermal contact with the cryostat bottom cold finger via $\mathrm{Cu}-\mathrm{Be}$ springs on the SQUID holder (see Fig.5a). Moreover, the excitation coil of the ac magnetic field is located on the movable DAC holder that is allowed to move with a micrometer accuracy along the three axes plus a rotation around the vertical directions to guarantee the alignment of the DAC respect to the light beam (Fig.5b). The whole sample insert is placed inside the cryostat by a load-lock system, to allow changing the pressure outside the cryostat while keeping it at LHe temperature. The sample between the anvils will be set at the center of the dc magnetic field along the light path while the gradiometer, connected by a flexible superconducting wire to the SQUID, is in thermal contact with the insert and the ac drive coil placed around the DAC. The system has been designed in order to host the SQUID, thanks also to a magnetic $\mathrm{Nb}_{3} \mathrm{Sn}$ shield cylinder, in a low magnetic field region $(<100$ Gauss) in the bottom section of the insert. The read-out of the ac signals from the SQUID electronics will be performed by a multi-harmonic lock-in. To be able to control and adjust in an easy and accurate way the sample temperature inside the DAC we will use an external laser $(\lambda=532 \mathrm{~nm})$.

E) SINBAD beamline and FTIR spectroscopy

Nowadays it is well recognized that because of its high brilliance, IR synchrotron radiation sources are ideal to perform high-pressure investigations on small samples such as those contained inside a DAC. SINBAD (Synchrotron Infrared Beamline At DAФNE) is an IR beamline operational since 2001 in the DAФNE-Light laboratory at DAФNE (Double Annular $\Phi$-factory for Nice Experiments), the Frascati electron-positron collider working in topping up mode at an energy of $0.51 \mathrm{GeV}$ per beam with a maximum beam current $>2 \mathrm{~A}$. With a bending magnet radius of $1.4 \mathrm{~m}$, this source is characterized by a critical energy of $208 \mathrm{eV}$ really optimized for low energy applications. As a consequence SINBAD was designed, assembled and commissioned to work at IR wavelengths from about 10 up to $12000 \mathrm{~cm}^{-1}$. SINBAD has been designed by ray-tracing simulations and its optical layout consists of six mirrors that first focus the radiation and than transfer the source image as a collimated beam to the experimental area. Therein a BRUKER Equinox 55 interferometer working in a vacuum chamber coupled to a BRUKER Hyperion 3000 microscope are available for FTIR experiments [9]. The SINBAD performances are suitable to work with small samples and or DACs and recently, in addition to other HP 
experiments, the first far-infrared absorption spectra of $\mathrm{La}_{1-\mathrm{x}} \mathrm{Ca}_{\mathrm{x}} \mathrm{MnO}_{3-\mathrm{x}}$ manganite samples at pressures up to 10 GPa have been collected using the IR synchrotron radiation emission. Data showed that pressure promotes partial metallization at room temperature through a strong reduction of the insulating gap [10].

The PRESS-MAG-O apparatus was designed to perform FTIR spectroscopy in transmission or reflection mode. Indeed, a parallel beam coming out by the interferometer is focused to the sample inside the DAC, using one of the four lateral ports equipped by a wedged diamond window to allow transmission of both visible and IR light. Focusing will be achieved by a $\mathrm{ZnSe}$ or by $\mathrm{KBr}$ lenses to work in the mid-IR or in the far-IR range, respectively. Simulations are in progress to replace lenses with Cassegrain objectives designed to this purpose. The transmitted beam will exit through a second diamond wedged window placed on the opposite lateral port and then focused to the detector position by a symmetrical optical component. The focusing lenses have a central hole to allow the beam of the laser to reach the sample following the same path of the IR light. As outlined in Fig.6, the laser will be highly focused by a lens concentric to the IR focusing lens and aligned independently to achieve its optimal focusing condition. For experiments performed in the reflection geometry, as in the microscope optical layout, the beam will be collected by half of the optical components used for the incoming beam in the transmission geometry.

\section{CONCLUSION}

When completed, PRESS-MAG-O will represent a unique and extremely versatile instrument capable to cover wide ranges of dc magnetic field $(\leq 8 \mathrm{~T})$, temperature $(4.2-200 \mathrm{~K})$ and pressure $(0-20 \mathrm{GPa})$, allowing concurrent or simultaneous FTIR spectroscopy and ac magnetic multi-harmonic susceptibility measurements $(10 \mathrm{~Hz}-20 \mathrm{KHz})$. This instrument is based on a cryostat for temperature measurements and an ac micro-gradiometer to probe the magnetic response of a sample contained inside a Cu-Be DAC. Both magneto-optical experiments vs. pressure and vs. magnetic field in a dc high field magnet will be possible using the IR synchrotron radiation of SINBAD, the beamline operational at Frascati. We expect this device will be operational in 2008 as an invaluable tool to perform systematic high pressure, high magnetic field experiments in a wide temperature range for forefront condensed matter researches.

\section{ACKNOWLEDGMENTS}

We thank all members of the Solid State Laser Laboratory of ENEA at Frascati for their hospitality and invaluable support during the laser heating tests. A special thank is also due to F. Tabacchioni (INAF) and the technical staff of the DAФNELight laboratory of the LNF.

\section{REFERENCES}

[1] K. Shimizu, H. Ishikawa, D. Takao, T. Yagi, K. Amaya, Nature 419 (2002) 597.

[2] V. Struzhkin, R..J. Hemley, H. Mao, Y.A. Timofeev, Nature 390 (1997) 382.

[3] J..J. Hamlin, V.G. Tissen, J.S. Schilling, Phys. Rev. B. 73 (2006) 094522.

[4] I. Kézsmárki, Sz. Csonka, H. Berger, L. Forró, P. Fazekas, G. Mihály, PRB 63 (2001) 0081106(R).

[5] Awadhesh Mani, A. Bharathi, and Y. Hariharan PRB 63 (2001) 115103.

[6] Y.A. Timofeev, A.N. Utyuzh, Instr. and Experim. Tech. 4 (2005) 550.

[7] E. Sterer, M.P. Pasternak, R.D. Taylor, Rev. Sci. Instrum., 61 (1990) 1117.

[8] R. Rosenbaum, S. Hsu, J. Chen, Y. Lin, J. Lin, J. Phys.: Cond. Matt. 13 (2001) 10041.

[9] M. Cestelli Guidi, M. Piccinini, A. Marcelli, A. Nucara, P. Calvani and E. Burattini, J. Opt. Soc. Amer. A 22 (2005) 2810.

[10] A. Sacchetti, M. Cestelli Guidi, E. Arcangeletti, A. Nucara, P. Calvani, M. Piccinini, A. Marcelli and P. Postorino, Phys. Rev. Lett. 96 (2006) 035503. 


\section{FIGURE CAPTIONS}

Fig. 1. Photo of the $8 \mathrm{~T}$ superconducting magnet with its radial access ports before the installation.

Fig. 2. Photographs of the magneto-optical cryostat during the assembly phase (top panels) of the magnet (bottom left panel) and an internal side view of the entire system (bottom right panel).

Fig. 3. Photos of two different views of the amagnetic CuBe DAC.

Fig. 4. Layout of the planar ac micro-gradiometer designed to collect the magnetic signal inside the DAC (top), photograph of the pick-up coils patterned on a mono-Si wafer (bottom).

Fig. 5. a) the fixed component of the sample insert with the SQUID/magnetic screen system at the bottom; b) layout of the $\mathrm{X}-\mathrm{Y}-\mathrm{Z}-\Theta$ movable part of the DAC holder.

Fig. 6. Optical layout for light spectroscopies. 


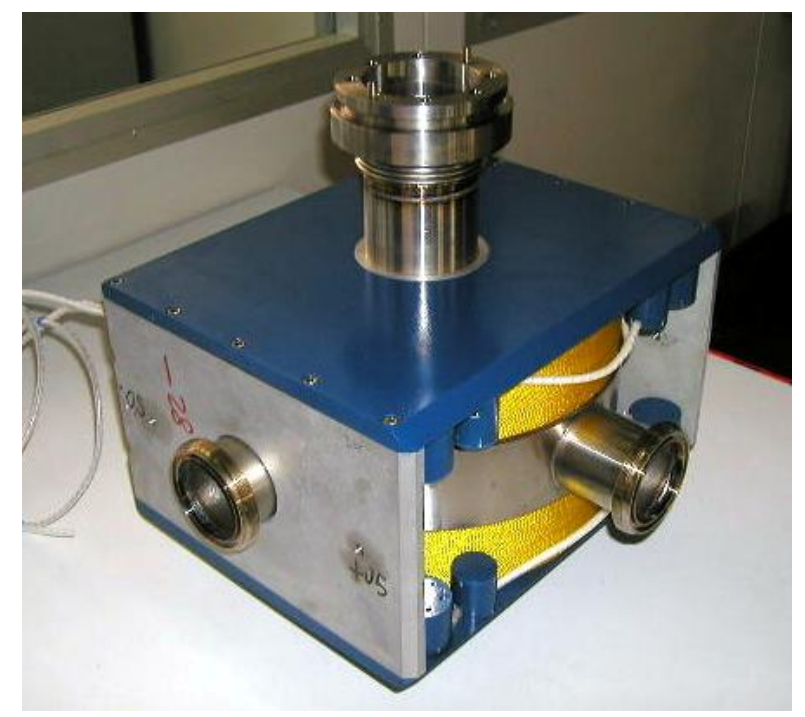

Fig. 1. Photo of the $8 \mathrm{~T}$ superconducting magnet with its radial access ports before the installation. 

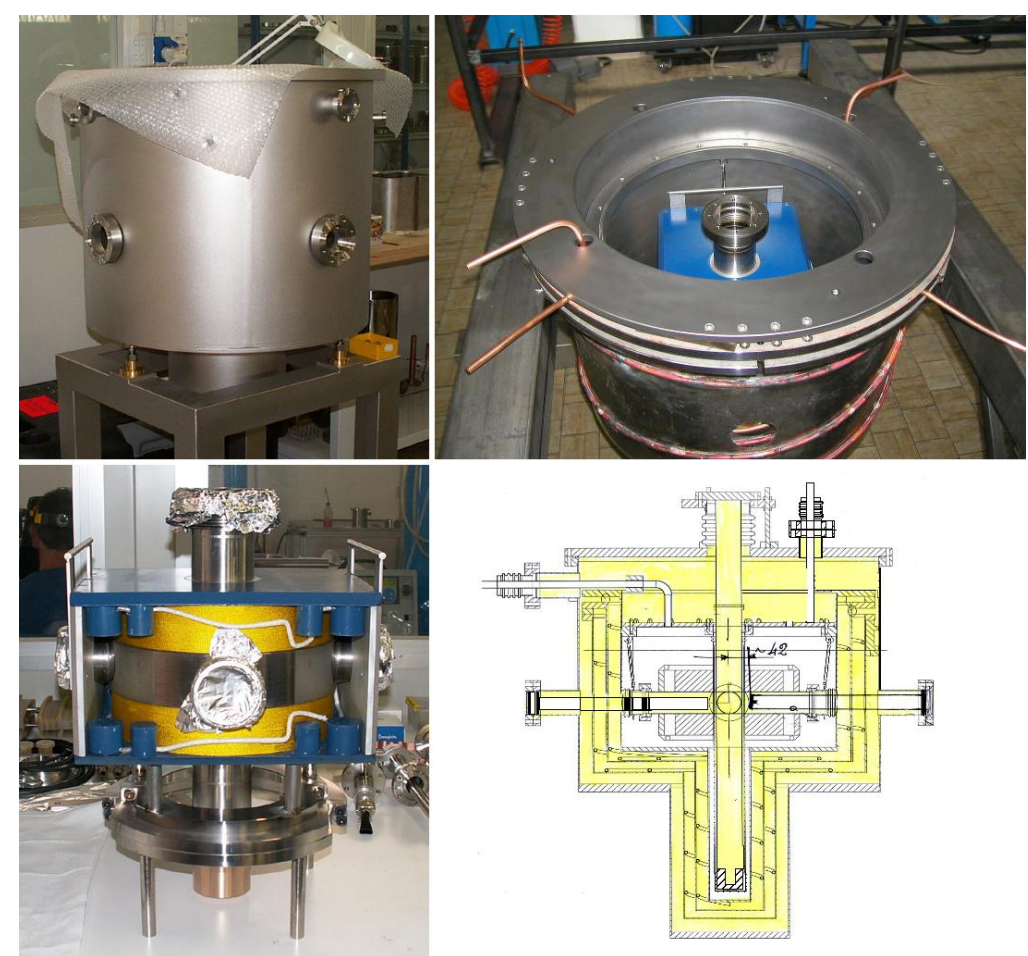

Fig. 2. Photographs of the magneto-optical cryostat during the assembly phase (top panels) of the magnet (bottom left panel) and an internal side view of the entire system (bottom right panel). 


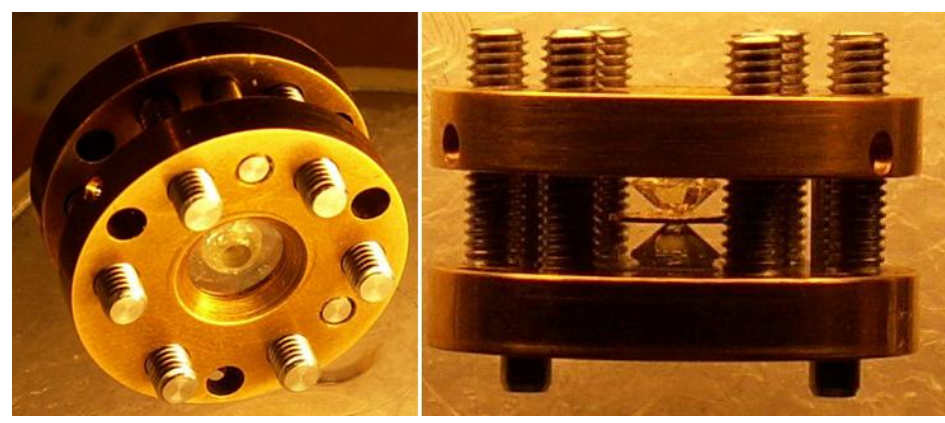

Fig. 3. Photos of two different views of the amagnetic CuBe DAC. 


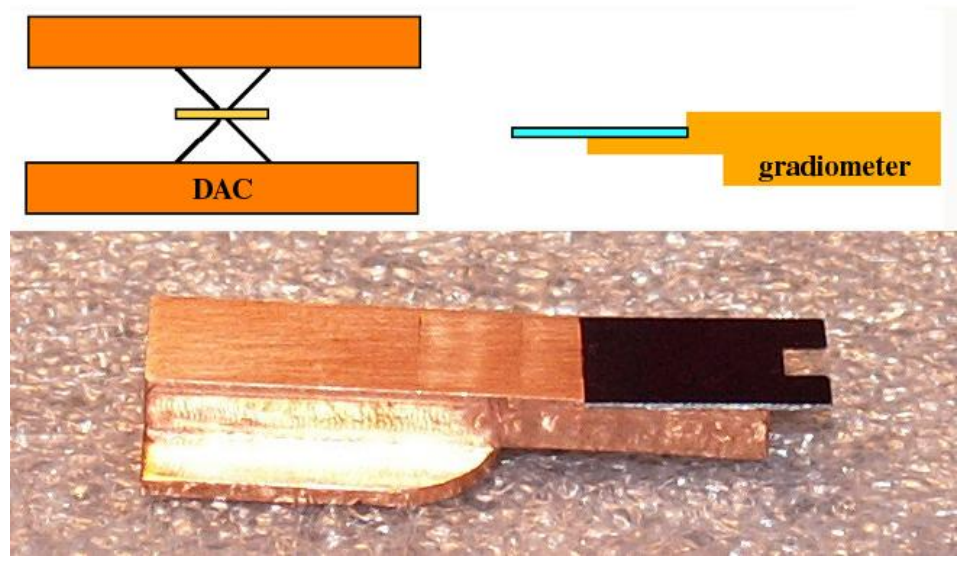

Fig. 4. Layout of the planar ac micro-gradiometer designed to collect the magnetic signal inside the DAC (top), photograph of the pick-up coils patterned on a mono-Si wafer (bottom). 


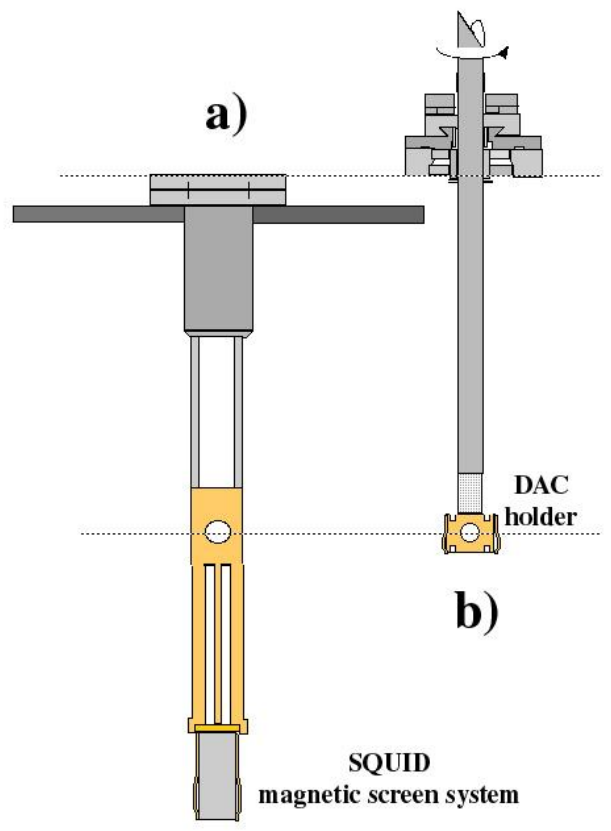

Fig. 5. a) The fixed component of the sample insert with the SQUID/magnetic screen system at the bottom; b) Layout of the X-Y-Z- $\Theta$ movable part of the DAC holder. 


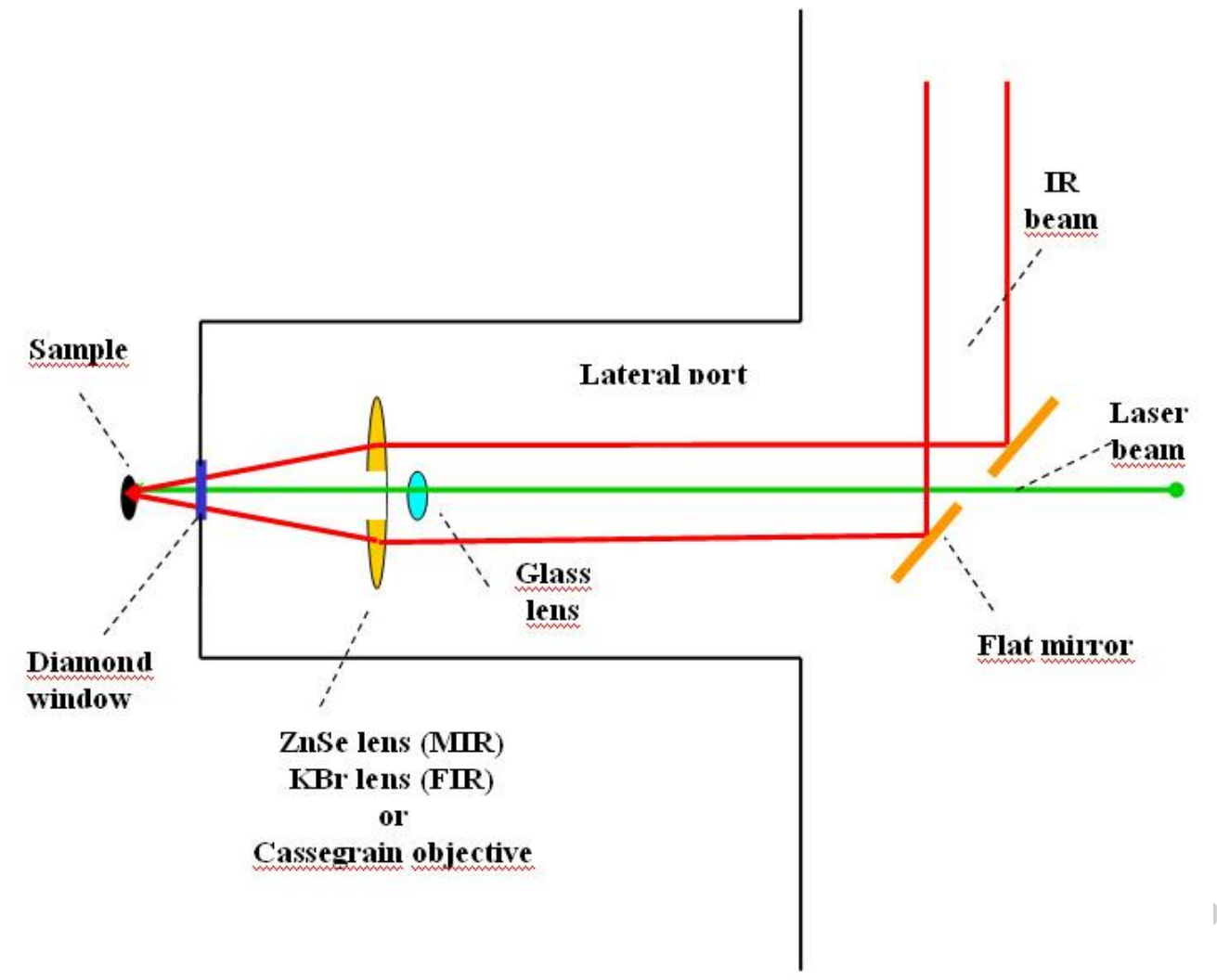

Fig 6. Optical layout for light spectroscopies. 